\title{
Numerical Technique to Increase the Accuracy of theADI Method in two Dimensional Equations
}

\author{
Ass. prof. Dr. FadhelSubhiFadhel , MeladJameelHmood \\ (Department of Mathematics, College of Science/Al-Nahrian University, Iraq) \\ (Department of Mathematics, College of Science/Al-Nahrian University, Iraq)
}

\begin{abstract}
Alternative Direction Iteration method for solving Partial Differential Equation by using the forth order finite difference method in time and space which will be still consist and unconditional stable.
\end{abstract}

Keywords: Accuracy of ADI method, Finite Difference Method, Partial differential Equation

\section{Introduction}

Alternating Direction Implicit (ADI) method was suggestedfirst by Peaceman and Rachford in the mid50s of the last century for solving systems of algebraic equations in two dimension, which results from the finite difference discretization method for solving PDEs [1]. From iterative method's perspective, the ADI method may be considered as a special relaxation method, where a big system is simplified into a number of smaller sub-systems, such that each of them may be solved efficiently and the solution of the whole system is got from the solutions of the sub-systems in an iterative method [2]. In classical (ADI) method, a second order (centered with time and backward with space) finite difference formulation usually used to obtain a scheme, the formulation obtained in this way will be unconditionally stable with a consistent condition which will be a sufficient for a method convergent.

\section{Implementation of The ADI Method}

Consider two dimensional diffusion equations [3]:

$$
\mathrm{u}_{\mathrm{t}}=\mathrm{u}_{\mathrm{xx}}+\mathrm{u}_{\mathrm{yy}} \mathrm{t} \in(0, \infty] \quad,(\mathrm{x}, \mathrm{y}) \in \mathrm{D}
$$

where $\mathrm{D}=\left(0, \mathrm{l}_{1}\right) \times\left(0, \mathrm{l}_{2}\right)$, with initial condition:

$$
\mathrm{u}(\mathrm{x}, \mathrm{y}, 0)=\mathrm{u}_{0}(\mathrm{x}, \mathrm{y}),(\mathrm{x}, \mathrm{y}) \in \mathrm{D}
$$

and the boundary conditions are at along $\partial \mathrm{D}$.

The finite difference formula:

$$
\begin{aligned}
& u_{t}=\frac{u_{i, j}^{n+1}-u_{i, j}^{n}}{\Delta t} \\
& u_{x x}=\frac{-u_{i+2}^{n}+16 u_{i+1}^{n}-30 u_{i}^{n}+16 u_{i-1}^{n}-u_{i-2}^{n}}{(\Delta x)^{2}} \ldots \ldots \ldots \\
& u_{y y}=\frac{-u_{j+2}^{n}+16 u_{j+1}^{n}-30 u_{j}^{n}+16 u_{j-1}^{n}-u_{j-2}^{n}}{(\Delta y)^{2}} \ldots \ldots
\end{aligned}
$$

This two formulation for $\mathrm{u}_{\mathrm{xx}}$ and $\mathrm{u}_{\mathrm{yy}}$ was the centeral differences with $\mathrm{O}(\mathrm{x})^{4}$ and $\mathrm{O}(\mathrm{y})^{4}$. Replacing both sides in diffusion equation by their central difference approximation, to get:

$$
\frac{u_{i, j}^{n+1}-u_{i, j}^{n}}{\Delta t}=\frac{-u_{i+2, j}^{n}+16 u_{i+1, j}^{n}-30 u_{i, j}^{n}+16 u_{i-1, j}^{n}-u_{i-2, j}^{n}}{(\Delta x)^{2}}+\frac{-u_{i, j+2}^{n}+16 u_{i, j+1}^{n}-30 u_{i, j}^{n}+16 u_{i, j-1}^{n}-u_{i, j-2}^{n}}{(\Delta y)^{2}}
$$

Then, after considering $\Delta \mathrm{x}=\Delta \mathrm{y}=\mathrm{h}$, and simplifying equation (5), it will become:

$$
\begin{aligned}
u_{i, j}^{n+1}-u_{i, j}^{n}= & \frac{\Delta t}{h^{2}}\left[\left(-u_{i+2, j}^{n}+16 u_{i+1, j}^{n}-30 u_{i, j}^{n}+16 u_{i-1, j}^{n}-u_{i-2, j}^{n}\right)\right. \\
& \left.+\left(-u_{i, j+2}^{n}+16 u_{i, j+1}^{n}-30 u_{i, j}^{n}+16 u_{i, j-1}^{n}-u_{i, j-2}^{n}\right)\right]
\end{aligned}
$$


In the ADI method formulation, equation (6) will be rearranged by taking two time steps as follows:

$$
\begin{aligned}
u_{i, j}^{n+1}-u_{i, j}^{n}= & r\left[\left(-u_{i+2, j}^{n}+16 u_{i+1, j}^{n}-30 u_{i, j}^{n}+16 u_{i-1, j}^{n}-u_{i-2, j}^{n}\right)\right. \\
+ & \left.\left(-u_{i, j+2}^{n+1}+16 u_{i, j+1}^{n+1}-30 u_{i, j}^{n+1}+16 u_{i, j-1}^{n+1}-u_{i, j-2}^{n+1}\right)\right] \\
u_{i, j}^{n+2}-u_{i, j}^{n+1}= & r\left[-u_{i+2, j}^{n+2}+16 u_{i+1, j}^{n+2}-30 u_{i, j}^{n+2}+16 u_{i-1, j}^{n+2}-u_{i-2, j}^{n+2}\right) \\
& \left.+\left(-u_{i, j+2}^{n+1}+16 u_{i, j+1}^{n+1}-30 u_{i, j}^{n+1}+16 u_{i, j-1}^{n+1}-u_{i, j-2}^{n+1}\right)\right]
\end{aligned}
$$

Simplify equations (7)and(8)with some rearrangement:

$$
\begin{aligned}
u_{i, j}^{n+1}-30 r u_{i, j}^{n+1}= & u_{i, j}^{n}-30 r u_{i, j}^{n}+r\left(-u_{i+2, j}^{n}+16 u_{i+1, j}^{n}+16 u_{i-1, j}^{n}-u_{i-2, j}^{n}\right) \\
& +r\left(-u_{i, j+2}^{n+1}+16 u_{i, j+1}^{n+1}+16 u_{i, j-1}^{n+1}-u_{i, j-2}^{n+1}\right) \ldots \ldots \ldots(9) \\
u_{i, j}^{n+2}-30 r u_{i, j}^{n+2}= & u_{i, j}^{n+1}-30 r u_{i, j}^{n+1}+r\left(-u_{i+2, j}^{n+2}+16 u_{i+1, j}^{n+2}+16 u_{i-1, j}^{n+2}-u_{i-2, j}^{n+2}\right) \\
& +r\left(-u_{i, j+2}^{n+1}+16 u_{i, j+1}^{n+1}+16 u_{i, j-1}^{n+1}-u_{i, j-2}^{n+1}\right) \ldots \ldots \ldots(10) \\
(1-30 r) u_{i, j}^{n+1}= & (1-30 r) u_{i, j}^{n}+r\left(-u_{i+2, j}^{n}+16 u_{i+1, j}^{n}+16 u_{i-1, j}^{n}-u_{i-2, j}^{n}\right) \\
& +r\left(-u_{i, j+2}^{n+1}+16 u_{i, j+1}^{n+1}+16 u_{i, j-1}^{n+1}-u_{i, j-2}^{n+1}\right) \ldots \ldots \ldots(11) \\
(1-30 r) u_{i, j}^{n+2}= & (1-30 r) u_{i, j}^{n+1}+r\left(-u_{i+2, j}^{n+2}+16 u_{i+1, j}^{n+2}+16 u_{i-1, j}^{n+2}-u_{i-2, j}^{n+2}\right) \\
+ & r\left(-u_{i, j+2}^{n+1}+16 u_{i, j+1}^{n+1}+16 u_{i, j-1}^{n+1}-u_{i, j}^{n+1}\right) \ldots \ldots \ldots(12)
\end{aligned}
$$

For $\mathrm{i}, \mathrm{j}=1,2, \ldots, \mathrm{n}$ equations (11) and (12) will used alternatively and the resulting systems will be tridiagonal that may be solved easily.

\section{Conclusion}

In this paper, we have obtained a new formula of the (ADI) method depending on central forth order finite difference formula which will be a forth order of time and space for more accuracy .

\section{References}

[1] Douglas Jim \&Peasman D.W., Numerical Solution for the Two Dimensional Heat Flow Problem, AM., Inst., Chemical Eng. Dept., J.1 505-512,1955.

[2] AL-SaifA.S.Jand AL-Kanani M.J, Alternating Direction Implicit Formulation of the Differential Quadrature Method for Solving the Unsteady State Two Dimensional Convection-Diffusion Equation,Gen. Math., Vol.7, No.1, pp.41-50, ISSN 2219-7184, November 2011

[3] Tristan S. Ursell,The Diffusion Equation A Multi-dimensional Tutorial, Department of Applied Physics, California Institute of Technology, Pasadena, , October 2007 